\title{
The neural correlates of conceptual and perceptual false recognition
}

\author{
Rachel J. Garoff-Eaton, ${ }^{1}$ Elizabeth A. Kensinger, ${ }^{2,3,4}$ and Daniel L. Schacter ${ }^{1,3,5}$ \\ ${ }^{1}$ Department of Psychology, Harvard University, Cambridge, Massachusetts 02138, USA; ${ }^{2}$ Department of Psychology, \\ Boston College, Chestnut Hill, Massachusetts 02467, USA; ${ }^{3}$ Athinoula A. Martinos Center for Biomedical Imaging, \\ Massachusetts General Hospital, Charlestown, Massachusetts 02129, USA
}

\begin{abstract}
False recognition, broadly defined as a claim to remember something that was not encountered previously, can arise for multiple reasons. For instance, a distinction can be made between conceptual false recognition (i.e., false alarms resulting from semantic or associative similarities between studied and tested items) and perceptual false recognition (i.e., false alarms resulting from physical similarities between studied and tested items). Although false recognition has been associated with frontal cortex activity, it is unclear whether this frontal activity can be modulated by the precise relationship between studied and falsely remembered items. We used event-related fMRI to examine the neural basis of conceptual compared with perceptual false recognition. Results revealed preferential activity in multiple frontal cortex regions during conceptual false recognition, which likely reflected increased semantic processing during conceptual (but not perceptual) memory errors. These results extend recent reports that different types of false recognition can rely on dissociable neural substrates, and they indicate that the frontal activity that is often observed during false compared with true recognition can be modulated by the relationship between studied and tested items.
\end{abstract}

Memory is subject to various kinds of distortions and illusions. These errors have been studied extensively by cognitive psychologists (e.g., Johnson et al. 1993; Roediger and McDermott 1995; Loftus 2003) and, more recently, by cognitive neuroscientists (e.g., Gonsalves et al. 2004; Kensinger and Schacter 2005; Okado and Stark 2005). For example, people sometimes claim to recognize things that they have never actually encountered (e.g., mistaking a stranger for an old acquaintance). This phenomenon, known as false recognition, has been the focus of much research, and neuropsychological and neuroimaging research has begun to reveal the neural processes underlying these false endorsements (for review, see Schacter and Slotnick 2004).

For instance, multiple studies have compared the neural activity that accompanies true recognition of studied items and false recognition of related but new items (e.g., falsely recognizing "chair" after studying a list of items of furniture) (Schacter et al. 1996b; Johnson et al. 1997; Cabeza et al. 2001; Goldmann et al. 2003; Slotnick and Schacter 2004). These neuroimaging studies, as well as neuropsychological ones, have implicated the frontal cortex in false recognition. Increased neural activity in regions of the frontal cortex has been observed during false compared with true recognition (Schacter et al. 1996b, 1997a; Cabeza et al. 2001; Goldmann et al. 2003; Slotnick and Schacter 2004), and patients with frontal lobe damage can show increased levels of false recognition (e.g., Parkin et al. 1996; Schacter et al. 1996a; Swick and Knight 1999; for review, see Schacter and Slotnick 2004). Although these results suggest that the frontal lobes are involved in memory errors, they do not indicate whether frontal activity during false recognition is modulated by the precise relationship between the studied and the falsely remembered items. For instance, within related false recognition, one can make the distinction between conceptual false recognition (i.e., false alarms resulting from semantic or associative similarities

\footnotetext{
Corresponding authors.
}

${ }^{4}$ E-mail Elizabeth.kensinger.1@bc.edu; fax (617) 552-0523.

5E-mail dls@wjh.harvard.edu; fax (617) 496-3122.

Article is online at http://www.learnmem.org/cgi/doi/10.1101/lm.695707. between studied and tested items) and perceptual false recognition (i.e., false alarms resulting from perceptual [e.g., visual, phonological] similarities between studied and tested items).

On the one hand, frontal activity during false recognition has been interpreted as reflecting monitoring processes, where falsely recognized items require increased retrieval effort or postretrieval evaluation (e.g., Schacter et al. 1996b; Cabeza et al. 2001; Goldmann et al. 2003; for review, see Schacter and Slotnick 2004). Under this view of frontal lobe function, one might predict that conceptual and perceptual false recognition would rely on similar levels of frontal lobe activity, since these monitoring processes should not necessarily be affected by information type. Consistent with that prediction, studies utilizing conceptually related stimuli (e.g., Schacter et al. 1996b; Cabeza et al. 2001), as well as those utilizing perceptually related stimuli (e.g., Goldmann et al. 2003; Slotnick and Schacter 2004), have reported frontal cortex activity increases during false recognition compared with true recognition.

On the other hand, behavioral studies investigating conceptual and perceptual false recognition in older adults and children have provided preliminary evidence that these two types of false alarms may rely differentially on frontal lobe function. Specifically, healthy older adults, compared with young adults, sometimes show a greater increase in false recognition for conceptually related items than for items that only share perceptual features with studied items (Koutstaal et al. 2003; but see Budson et al. 2003). Furthermore, distinct developmental trends are found for conceptual and perceptual false recognition; between the ages of 8 and $13 \mathrm{yr}$, conceptual false recognition rates increase, while perceptual false recognition rates decrease (Holliday and Weekes 2006). Because the frontal lobes undergo a particularly large amount of structural and functional change both during childhood (Diamond 2002) and during the aging process (Anderson and Craik 2000; Raz 2000), these findings raise the possibility that conceptual and perceptual false recognition are differentially reliant on frontal lobe processes. Therefore, the major changes in the frontal lobes during development and aging may 
underlie the differential effect of age on conceptual versus perceptual false recognition (for evidence that conceptual recognition can be disproportionately impaired compared with perceptual recognition, see also Schacter et al. 1997b, Budson et al. 2003). Moreover, it makes good sense to postulate that conceptual false recognition would be more reliant on frontal processes than perceptual false recognition; frontal activity can reflect semantic processing (e.g., semantic retrieval, semantic elaboration, semantic relational memory) (Wagner et al. 2001; Kirchhoff et al. 2005; Prince et al. 2005), which would presumably accompany conceptual false recognition more often than perceptual false recognition.

Although no prior study has compared the neural processes associated with conceptual and perceptual false recognition, recent work has shown that different types of false recognition can rely on distinct neural substrates. For example, the neural correlates of false recognition can vary depending on the context in which one believes to have studied an item previously (Kahn et al. 2004). Moreover, related false recognition (i.e., "old" responses to related but new items) and unrelated false recognition (i.e., "old" responses to unrelated, new items) have been shown to rely on distinct patterns of neural activity (Garoff-Eaton et al. 2006), which indicates that the relationship between a falsely remembered item and previously studied items must be considered when exploring the neural basis of memory errors. Therefore, it is plausible that the precise nature of the relationship (i.e., conceptual vs. perceptual) between falsely remembered and studied items could also affect the neural correlates of memory errors.

The primary goal of the present study was to examine whether frontal activity would be equally strong during conceptual and perceptual false recognition or whether the frontal cortex would be differentially engaged during conceptual false recognition. To distinguish between these alternatives, we conducted an event-related fMRI study that compared the neural activity associated with conceptual memory errors with the activity associated with perceptual memory errors. During study, participants viewed a series of word triplets. The words in each triplet were related to each other either conceptually (i.e., based on their meaning) or perceptually (i.e., based on their spelling and sound). (It should be noted that we use the term "perceptual" to refer to both visual and phonological properties of the stimuli.) At test, participants viewed a series of single words and decided whether or not they had studied those words previously. These tested words included: (1) old, studied words from conceptual or perceptual triplets; (2) lure words that were related to studied conceptual or perceptual triplets; and (3) new, unrelated words (Fig. 1). Related false recognition occurred when participants endorsed lures as old, and the classification of these errors as either conceptual or perceptual was determined by the relationship of the lure to the related, studied triplet.

\section{Results}

\section{Behavioral results}

Participants were able to successfully discriminate between studied and nonstudied words on the recognition test (Table 1). Participants were significantly more likely to give either a "remember" or a "know" response to an old conceptual word (0.88) or an old perceptual word (0.74) than they were to give either of those responses to an unrelated new word $(0.37$; both $P<0.0001)$. Furthermore, participants were also significantly more likely to give either a "remember" or a "know" response to a conceptual lure (0.62) or a perceptual lure $(0.49)$ than they were to give those responses to an unrelated new word (both $P<0.0001$ ), indicating that the relatedness of the lures did affect participants' responses in both the conceptual and the perceptual conditions. It is im-
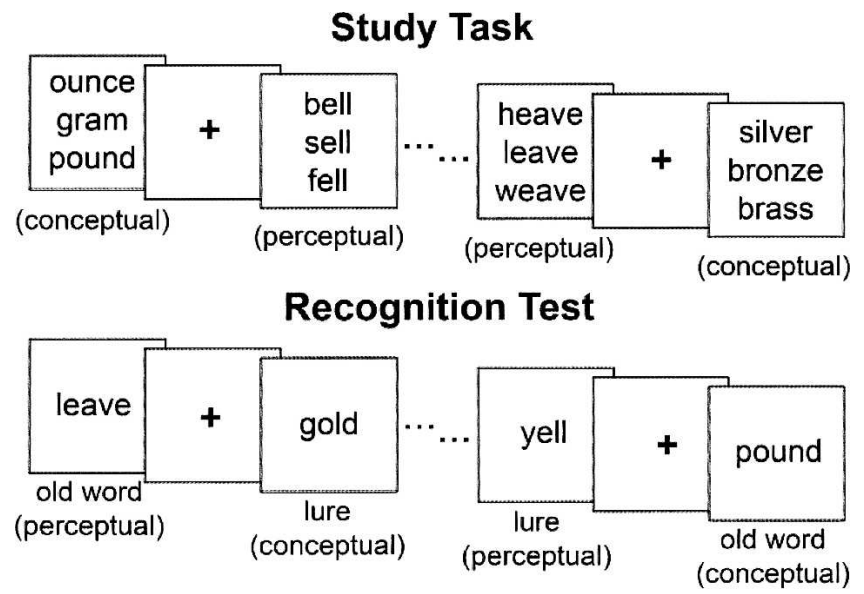

Figure 1. Schematic of the experimental procedure. During the study task, participants viewed word triplets that were either conceptually or perceptually related. Participants were instructed to decide how similar the words in each triplet were to each other while also remembering the words for the subsequent recognition test. During the recognition test, participants viewed five types of words: old conceptual words, old perceptual words, conceptual lures, perceptual lures, and unrelated new words. They gave a "remember," "know," or "new" response to each tested word. The condition in which words were tested was counterbalanced across participants by changing the word triplets shown during the study task (e.g., yell could be tested as a perceptual lure [shown here]; as a conceptual lure after studying shout, cry, squeal; as an old perceptual word after studying fell, bell, yell; as an old conceptual word after studying shout, yell, squeal; or as an unrelated new word after studying only unrelated triplets).

portant to note that the old conceptual and conceptual lure conditions elicited significantly more "remember" responses than the old perceptual and perceptual lure conditions, respectively (i.e., "remember"/old conceptual > "remember"/old perceptual, "remember"/conceptual lure > "remember"/perceptual lure; both $P<0.0005)$. These differences remained even when conditionalized scores were compared (i.e., proportion of "remember" responses, given that an item was not called "new" ["remember"/ ("remember" + "know")]; both $P<0.0005)$. These results replicate earlier findings that "remember" responses tend to accompany conceptual false recognition more often than perceptual false recognition (Schacter et al. 1997b; Watson et al. 2003). Thus, given that remembering and knowing can rely on distinct patterns of neural activity (Wheeler and Buckner 2004), we chose to limit our fMRI analysis to "remember" responses so that our neuroimaging comparisons of conceptual versus perceptual memory processing would not be contaminated by differences in remembering versus knowing. (Note that because conceptual true and false recognition were accompanied by "remember" responses more often than perceptual true and false recognition, the perceptual conditions are composed of fewer trials than the conceptual conditions.) "Remember" responses also are relatively straightforward to interpret (e.g., a "remember" response to a lure item is a clear indication of a false memory; the person is claiming that they remember the instance in which they were presented with the word), whereas "know" responses may be more likely to include guesses or responses based upon familiarity with related studied items. Importantly, "remember" responses were given to conceptual and perceptual old words and lures more often than they were given to unrelated new words (all $P<0.001)$. Because most participants gave an insufficient number of "remember" responses to unrelated new words (i.e., unrelated false recognition), we did not include that condition in any fMRI analyses. Furthermore, the set of 14 participants with a 
Table 1. At the top, proportion (mean [SE]) of items given a "remember," "know," or "new" response as a function of item type; at the bottom, response latencies (mean [SE]) associated with each response type as a function of item type.

\begin{tabular}{lccccc} 
Response type & Old word (conceptual) & Old word (perceptual) & Lure (conceptual) & Lure (perceptual) & New word (unrelated) \\
\hline $\begin{array}{l}\text { Accuracy } \\
\text { "Remember" }\end{array}$ & $0.71(0.05)$ & $0.37(0.04)$ & $0.35(0.03)$ & $0.17(0.02)$ & $0.09(0.02)$ \\
"Know" & $0.17(0.03)$ & $0.36(0.04)$ & $0.27(0.03)$ & $0.33(0.02)$ & $0.28(0.02)$ \\
$\quad$ "New" & $0.10(0.03)$ & $0.26(0.02)$ & $0.37(0.03)$ & $0.49(0.02)$ & $0.62(0.03)$ \\
Response latencies (msec) & $1267(75)$ & $1243(62)$ & $1434(58)$ & $1495(94)$ & $1735(98)$ \\
Remember" & $1798(133)$ & $1766(91)$ & $1740(105)$ & $1694(105)$ & $1666(109)$ \\
"Know" & $1633(115)$ & $1732(107)$ & $1676(87)$ & $1572(88()$ & $1526(76)$ \\
"New" & & & &
\end{tabular}

sufficient number of "remember" responses to studied words and lures was not the same set of participants with a sufficient number of "know" responses to studied words and lures; therefore, we will not report any fMRI comparisons between "remember" and "know" responses here.

Focusing on true and false recognition (i.e., "remember" responses to old words and lures), participants were faster to respond to conceptual or perceptual old words than they were to respond to conceptual or perceptual lures, respectively (i.e., conceptual true recognition < conceptual false recognition, perceptual true recognition < perceptual false recognition, based on average median response latencies; both $P<0.005)$. Critically, there was no difference in participants' median response latencies to conceptual versus perceptual true recognition (old conceptual vs. old perceptual) or false recognition (conceptual lure vs. perceptual lure; both $P>0.2$ ) (see Table 1).

\section{fMRI results}

A frontoparietal network of brain regions was active during both conceptual and perceptual true recognition ("remember"/old conceptual and "remember"/old perceptual, respectively) compared with correct rejections ("new"/unrelated new), which is consistent with prior reports of neural activity patterns accompanying remembering (e.g., Buckner et al. 1998; Buckner and Wheeler 2001). A similar network of frontal and parietal regions was also active during both conceptual and perceptual false recognition ("remember"/conceptual lure and "remember"/ perceptual lure, respectively) compared with correct rejections. These results are consistent with past reports of overlapping activity associated with true and false recognition in frontal and parietal regions (e.g., Cabeza et al. 2001; Slotnick and Schacter 2004; Garoff-Eaton et al. 2006), and they indicate that similarities do exist between conceptual and perceptual memory processing. Moreover, consistent with previous reports of frontal cortex activity observed during false recognition (e.g., Schacter et al. 1996b, 1997a; Cabeza et al. 2001; Goldmann et al. 2003; Slotnick and Schacter 2004), false compared with true recognition, regardless of information type ("remember"/conceptual lure and perceptual lure > "remember"/old conceptual and old perceptual), was associated with activity increases in multiple frontal cortex regions, including the middle, medial, and inferior frontal gyri (Table 2). By contrast, we did not observe any activity increases during all true recognition compared with all false recognition ("remember"/old conceptual and old perceptual > "remember"/ conceptual lure and perceptual lure).

Although these analyses suggest that at least some memory processes are unaffected by the type of information being retrieved, we were particularly interested in differential activity associated with conceptual versus perceptual memory processing. Collapsing across true and false recognition, conceptual memory compared with perceptual memory ("remember"/old conceptual and conceptual lure > "remember"/old perceptual and perceptual lure) was accompanied by activity increases in multiple regions, including the frontal (e.g., superior, middle, and medial frontal gyri) and parietal cortex (e.g., precuneus and superior parietal lobule) (see Table 2). By contrast, the opposite analysis ("remember"/old perceptual and perceptual lure > "remember"/old conceptual and conceptual lure) revealed activity increases during perceptual memory (compared with conceptual memory) in only two regions, the left inferior frontal gyrus (BA 44/9) and the left inferior parietal lobule (BA 40) (see Table 2).

Turning our focus to false recognition, we used a masking function (see Materials and Methods) to identify neural activity increases associated with false (compared with true) recognition that were unique to either conceptual or perceptual information. First, we identified those regions that were more active during false than true conceptual recognition but were not more active during false than true perceptual recognition (i.e., those regions

Table 2. Neural activity observed during false vs. true recognition (regardless of information type) and conceptual vs. perceptual recognition (regardless of accuracy)

\begin{tabular}{lcrrr}
\hline Region & BA & x & \multicolumn{1}{c}{ y } & z \\
\hline All false > all true & & & & \\
Left middle frontal gyrus & 9 & -46 & 29 & 30 \\
Left middle frontal gyrus & $8 / 9$ & -44 & 9 & 33 \\
Left inferior frontal gyrus & $45 / 46$ & -44 & 28 & 17 \\
Left inferior frontal gurus & $44 / 9$ & -46 & 5 & 24 \\
Left inferior frontal gyrus & 47 & -32 & 21 & -11 \\
Right medial frontal gyrus & $8 / 6$ & 16 & 31 & 39 \\
Left medial frontal gyrus & $9 / 8 / 6$ & -4 & 31 & 33 \\
Right anterior cingulate & $32 / 24$ & 8 & 30 & 19 \\
Right insula & 13 & 32 & 20 & 6 \\
All conceptual > all perceptual & & & & \\
Left superior frontal gyrus & 8 & -40 & 18 & 49 \\
Left middle frontal gyrus & 6 & -40 & 14 & 49 \\
Left medial frontal gyrus & $11 / 10$ & -10 & 50 & -11 \\
Left supramarginal gyrus & $40 / 39$ & -50 & -59 & 31 \\
Left cingulate gyrus & 31 & -10 & -55 & 29 \\
Left posterior cingulate & $23 / 30$ & -4 & -53 & 19 \\
Left superior parietal lobule & 7 & -40 & -70 & 46 \\
Left precuneus & 7 & -6 & -68 & 33 \\
Left/right precuneus & $23 / 31$ & -2 & -61 & 18 \\
Left precuneus & $31 / 7$ & -2 & -51 & 32 \\
Right precuneus & 31 & 2 & -65 & 29 \\
Left cuneus & $30 / 23$ & -6 & -60 & 9 \\
Right cuneus & 18 & 4 & -71 & 16 \\
Left cuneus & $18 / 19$ & -2 & -78 & 28 \\
Right lingual gyrus & 18 & 12 & -68 & 5 \\
Right middle occipital gyrus & 18 & 10 & -91 & 12 \\
Right pons & - & 14 & -40 & -28 \\
Left pons & - & -10 & -35 & -30 \\
Right cerebellum & - & 8 & -42 & -18 \\
Left cerebellum & - & -10 & -45 & -16 \\
All perceptual > all conceptual & & & & \\
Left inferior frontal gyrus & $44 / 9$ & -51 & 9 & 24 \\
Left inferior parietal lobule & 40 & -50 & -35 & 46 \\
& & & & \\
\hline & & &
\end{tabular}

BA refers to Brodmann area, and coordinated $(x, y, z)$ are reported in Talairach space. 
that were active in the "remember"/conceptual lure > "remember"/old conceptual contrast but were not active in the "remember"/perceptual lure > "remember"/old perceptual contrast). This analysis revealed widespread activity throughout the frontal cortex (BAs 6, 8, 9, 44, 45, 46, 47), as well as activity in the precentral gyrus and precuneus (Table 3; Fig. 2). By contrast, the reverse analysis (i.e., identifying those regions that were active in the "remember"/perceptual lure > "remember"/old perceptual contrast but were not active in the "remember"/conceptual lure > "remember"/old conceptual contrast) revealed only one cluster of activity in the caudate (see Table 3 ). Complementary analyses were performed to identify activity increases during true (compared with false) recognition that were unique to either conceptual or perceptual information. Only one cluster of activity spanning the left insula and claustrum $(\mathrm{x}=-36, \mathrm{y}=-18, \mathrm{z}=-1$; $\mathrm{BA}$ 13) was more active during true than false conceptual (but not perceptual) recognition ("remember"/old conceptual > "remember"/conceptual lure not "remember"/old perceptual > "remember"/perceptual lure), and no regions were more active during true than false perceptual (but not conceptual) recognition ("remember"/old perceptual > "remember"/perceptual lure not "remember"/old conceptual > "remember"/conceptual lure). It is unlikely that activity observed in these analyses was due to differences in effort, given that the same reaction time difference existed in both the included and the excluded contrast (i.e., activity associated with lengthier response latencies during false compared with true recognition should be present in both the contrast of interest and in the contrast used to create the mask, thus excluding activity associated only with longer reaction times from the final analysis).

To further investigate the differential activity observed in

Table 3. Neural activity observed during false recognition compared with true recognition for either conceptual (but not perceptual) information or perceptual (but not conceptual) information

\begin{tabular}{|c|c|c|c|c|}
\hline Region & BA & $x$ & $y$ & z \\
\hline \multicolumn{5}{|c|}{$\begin{array}{l}\text { Conceptual false }>\text { conceptual true } \\
\text { (not perceptual false }>\text { perceptual true) }\end{array}$} \\
\hline Left superior frontal gyrus & 8 & -6 & 14 & 47 \\
\hline Left superior frontal gyrus & 6 & -2 & 18 & 53 \\
\hline Left middle frontal gyrus & 9 & -44 & 9 & 33 \\
\hline Left middle frontal gyrus & 6 & -26 & 12 & 45 \\
\hline Left middle frontal gyrus & 6 & -38 & -2 & 44 \\
\hline Left middle frontal gyrus & 46 & -51 & 26 & 24 \\
\hline Right inferior frontal gyrus & $44 / 45$ & 55 & 11 & 22 \\
\hline Left inferior frontal gyrus & 45 & -48 & 35 & 6 \\
\hline Left inferior frontal gyrus & 47 & -48 & 36 & -9 \\
\hline Left inferior frontal gyrus & 47 & -32 & 23 & -10 \\
\hline Right medial frontal gyrus & 9 & 9 & 43 & 21 \\
\hline Right medial frontal gyrus & 9 & 4 & 44 & 33 \\
\hline Left medial frontal gyrus & 9 & -2 & 40 & 31 \\
\hline Left medial frontal gyrus & 8 & -4 & 27 & 41 \\
\hline Left medial frontal gyrus & 6 & -4 & 31 & 37 \\
\hline Right precentral gyrus & 6 & 28 & -19 & 54 \\
\hline Left precuneus & 7 & -6 & -62 & 51 \\
\hline Left/right midbrain & - & -2 & -16 & -4 \\
\hline \multicolumn{5}{|c|}{$\begin{array}{l}\text { Perceptual false }>\text { perceptual true } \\
\quad(\text { not conceptual false }>\text { conceptual true) }\end{array}$} \\
\hline Right caudate & - & 12 & -12 & 23 \\
\hline \multicolumn{5}{|c|}{$\begin{array}{l}\text { (Conceptual false }>\text { conceptual true) }>\text { (perceptual } \\
\text { false }>\text { perceptual true) }\end{array}$} \\
\hline Left inferior frontal gyrus & $45 / 47$ & -46 & 37 & 2 \\
\hline \multicolumn{5}{|c|}{$\begin{array}{l}\text { (Perceptual false }>\text { perceptual true) }>\text { (conceptual } \\
\quad \text { false }>\text { conceptual true) }\end{array}$} \\
\hline Right middle temporal gyrus & 21 & 55 & -48 & -3 \\
\hline
\end{tabular}

BA refers to Brodmann area, and coordinates $(x, y, z)$ are reported in Talairach space. the frontal cortex during false compared with true recognition of conceptual (but not perceptual) information, we extracted eventrelated timecourses from each cluster of frontal activity defined by the "remember"/conceptual lure > "remember"/old conceptual not "remember"/perceptual lure > "remember"/old perceptual analysis. (This analysis resulted in nine clusters of activity in the frontal cortex. More than nine frontal regions are listed in Table 3 because several clusters spanned more than one region or Brodmann area. Event-related timecourses were extracted from each cluster of activity rather than each region so that all clusters were spatially independent, a requirement for computing the joint probability reported here [see Materials and Methods].) Although the random effects analysis requires a larger difference in activity between false and true conceptual recognition than between false and true perceptual recognition, it does not require a specific relationship between conceptual false recognition and perceptual false recognition (i.e., event-related timecourses are necessary to determine whether activity observed during conceptual false recognition is greater than, less than, or equal to activity observed during perceptual false recognition in the frontal cortex regions defined by the random effects analysis). Critically, the event-related timecourses revealed that, in all nine clusters of frontal cortex activity, greater activity (as assessed at 4-8 sec after stimulus onset) was associated with conceptual false recognition than perceptual false recognition (see Fig. 2). This difference reached statistical significance in two regions of left middle frontal gyrus (both $P<0.05$ ). Importantly, the joint probability of observing this same pattern of differential activity across all nine of these clusters was significant (joint $P$-value $<0.005$ ). The same pattern of results was observed when event-related activity assessed at $6 \mathrm{sec}$ after stimulus onset was analyzed (joint $P$-value $<0.03$ ).

Additional analyses that did not rely on masking procedures were also performed in order to further explore neural activity that was modulated by both accuracy and information type. Specifically, an interaction analysis revealed that the left inferior frontal gyrus (BA 45/47) showed a greater difference in activity between conceptual false and true recognition than between perceptual false and true recognition (["remember"/conceptual lure $>$ "remember"/old conceptual] > ["remember"/perceptual lure > "remember"/old perceptual]) (see Table 3; Fig. 3). Event-related timecourses extracted from this active cluster revealed significantly greater activity associated with conceptual false recognition than perceptual false recognition $(P<0.04)$ (see Fig. 3 ). By contrast, activity in the right middle temporal gyrus (BA 21), but not in any frontal cortex regions, showed a greater difference in activity between perceptual false and true recognition than between conceptual false and true recognition (["remember"/ perceptual lure > "remember"/old perceptual] > ["remember"/ conceptual lure $>$ "remember"/old conceptual]) (see Table 3).

In a direct comparison of conceptual and perceptual false recognition (i.e., "remember"/conceptual lure vs. "remember"/ perceptual lure), conceptual false recognition (compared with perceptual false recognition) was accompanied by increased activity in left BA 39 spanning both the angular and middle temporal gyri $(\mathrm{x}=-40, \mathrm{y}=-61, \mathrm{z}=25)$, in the right inferior parietal lobule extending into the post-central gyrus $(x=55, y=-30$, $\mathrm{z}=20$; $\mathrm{BA} 40$ ), and in a region bordering the right inferior/ middle frontal gyri $(x=22, y=35, z=-5$; BA 47/11). By contrast, increased activity in only one cluster of activity bordering the left cingulate gyrus $(x=-20, y=-43, z=35$; BA 31) was observed during perceptual false recognition (compared with conceptual false recognition).

\section{Discussion}

The results from the current study indicate that although similarities between conceptual and perceptual memory processes ex- 


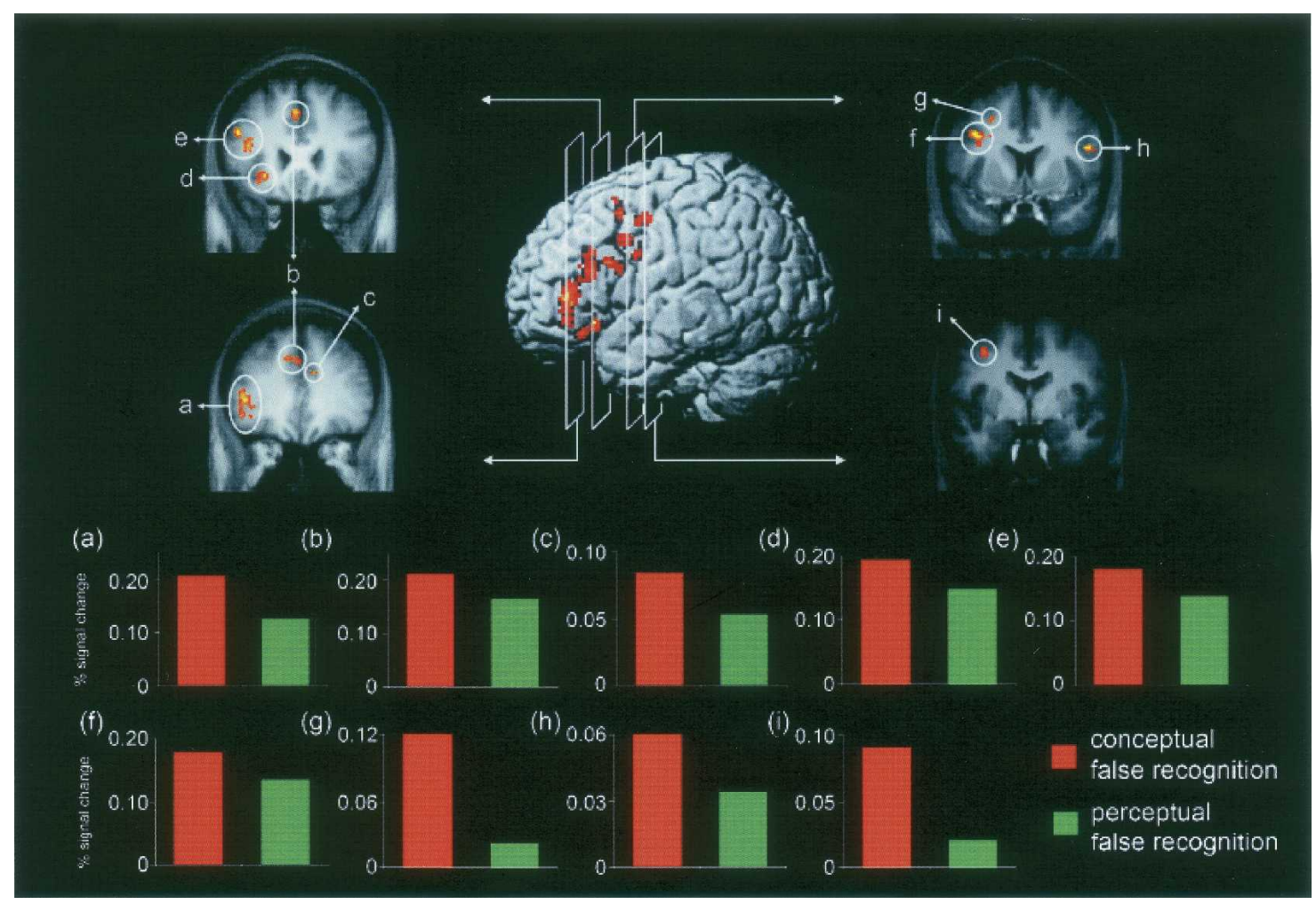

Figure 2. Neural activity associated with conceptual false compared with conceptual true recognition but not with perceptual false compared with perceptual true recognition ("remember"/conceptual lure > "remember"/old conceptual excluding activity in "remember"/perceptual lure > "remember"/old perceptual). In the center, activity identified by this analysis is projected onto the left lateral surface of a three-dimensional template brain. On each side, activity is projected onto coronal slices of the group mean anatomic image, and each of the nine clusters of frontal activity identified by this analysis is demarcated by a circle. In the bottom panel, event-related activity (4-8 sec after stimulus onset) extracted from these active clusters is shown. ( $a$, inferior frontal gyrus; $b$, medial/superior frontal gyri; $c$, medial frontal gyrus; $d$, inferior frontal gyrus; $e$, middle frontal gyrus; $f$, middle frontal gyrus; $g$, middle frontal gyrus; $h$, inferior frontal gyrus; $i$, middle frontal gyrus)

ist, the frontal cortex is preferentially active during conceptual false recognition. Specifically, similar standard patterns of neural activity (including activity in frontal and parietal regions) (Buckner et al. 1998; Buckner and Wheeler 2001) were observed during true and false recognition for both conceptual and perceptual information compared with correct rejections. Furthermore, consistent with past comparisons of true and false recognition (Schacter et al. 1996b, 1997a; Cabeza et al. 2001; Goldmann et al. 2003; Slotnick and Schacter 2004), increased frontal cortex activity accompanied false (compared with true) recognition regardless of information type. Importantly, subsequent analyses qualified that finding, indicating that the increased activity observed in certain frontal cortex regions during false recognition may be most pronounced when there is a conceptual (rather than a perceptual) relationship between the studied and the falsely recognized items. For instance, when the neural correlates of false and true recognition were compared separately for each information type, false recognition for conceptual (but not perceptual) information was supported by increased activity in multiple frontal cortex regions. In fact, event-related timecourses illustrated that in all of these frontal regions, greater activity accompanied conceptual than perceptual false recognition. By contrast, there was no indication of frontal cortex activity that supported perceptual rather than conceptual false recognition. Thus, our results suggest that conceptual false recognition is particularly reliant upon frontal lobe activity, and that the neural underpinnings of conceptual and perceptual false recognition are at least partially distinct.

These results complement previous behavioral research.
Specifically, our results suggest that frontal activity is preferentially involved in conceptual false recognition, which could explain the different effects of childhood development (Holliday and Weekes 2006) and aging (Koutstaal et al. 2003) on conceptual versus perceptual false recognition, given the pronounced frontal lobe changes that occur during these developmental periods (Anderson and Craik 2000; Raz 2000; Diamond 2002). In fact, using the same behavioral paradigm as was used in this fMRI

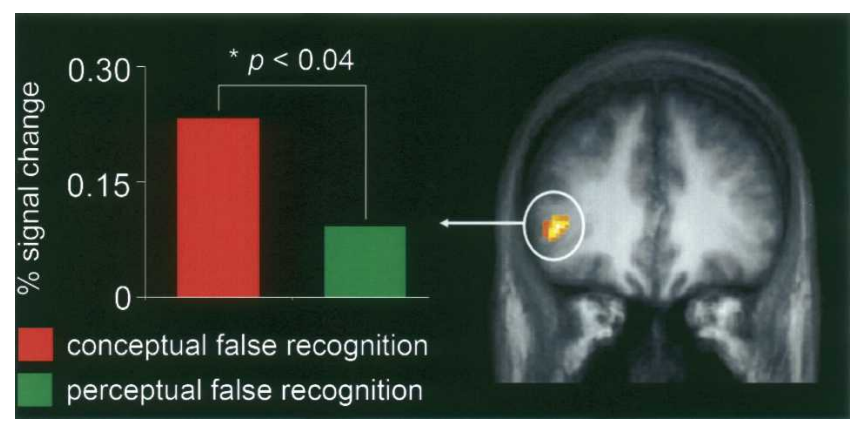

Figure 3. Interaction analysis identifying regions that show a greater difference in activity between conceptual false and true recognition than between perceptual false and true recognition (["remember"/conceptual lure $>$ "remember"/old conceptual] > ["remember"/perceptual lure > "remember"/old perceptual]). On the right, activity is projected onto a coronal slice of the mean anatomic image, and on the left, the eventrelated activity (4-8 sec after stimulus onset) extracted from the active cluster within the left inferior frontal cortex (BA 45/47) is shown. 
experiment, older adults compared with young adults show increased rates of conceptual false recognition, but equivalent rates of perceptual false recognition, perceptual true recognition, and conceptual true recognition (R.J. Garoff-Eaton, E.A. Kensinger, and D.L. Schacter, unpubl.). According to the results from the current study, it could be hypothesized that this differential effect of aging on conceptual and perceptual memory errors is due to altered frontal cortex activity in older adults, where conceptual false recognition is more reliant on frontal cortex activity and thus is more affected by the frontal lobe changes that accompany aging; further research would be necessary to test that hypothesis.

Our results indicate that the frontal cortex is preferentially engaged during conceptual compared with perceptual false recognition, but what cognitive processes does this frontal lobe activity reflect? Past interpretations of frontal activity during false recognition have often focused on post-retrieval monitoring processes (e.g., Schacter et al. 1996b; Cabeza et al. 2001; Goldmann et al. 2003; for review, see Schacter and Slotnick 2004). Under this view, the monitoring or verification processes that take place after retrieval has occurred (and that engage the prefrontal cortex) are more extensive and/or effortful when the retrieved information was never studied (i.e., during false recognition) than when it was studied previously (i.e., during true recognition). This interpretation is consistent with previous reports of increased prefrontal cortex activity during memory tasks that have heightened evaluation or monitoring demands (e.g., tasks requiring source or associative memory in addition to item memory) (Rugg et al. 1996; Henson et al. 1999; Rugg et al. 2003; Achim and Lepage 2005). When considering the role of post-retrieval monitoring in false recognition, one might assume that monitoring should occur regardless of the precise relationship between the studied and the falsely recognized items. For instance, it seems likely that monitoring would be required during both conceptual and perceptual false recognition to evaluate or verify the accuracy of the familiar information being retrieved. Indeed, some of the overlapping frontal cortex activity we observed during both conceptual and perceptual false recognition may reflect postretrieval monitoring; however, it is important to note that this overlapping activity was primarily left lateralized, and some have argued that it is the right (not left) prefrontal cortex that is preferentially involved in post-retrieval monitoring of familiarity (e.g., Dobbins et al. 2004) or evaluation/verification monitoring processes (e.g., Cabeza et al. 2003).

Furthermore, it seems unlikely that the primarily left lateralized regions found to be more active during conceptual than perceptual false recognition reflect post-retrieval monitoring alone. Instead, this frontal activity may reflect additional cognitive processes that are likely to be preferentially involved in conceptual memory. For instance, regions of the frontal cortex (especially the left inferior frontal cortex) are particularly active during successful semantic processing (Demb et al. 1995), word generation (Petersen et al. 1988), recovery of meaning/semantic retrieval (Wagner et al. 2001), verbal recall (Cabeza et al. 2003), semantic (compared with perceptual) relational memory (Prince et al. 2005), and semantic elaboration (Kirchhoff et al. 2005). In the current study, false recognition of a conceptual lure is likely to occur if a participant elaborates on the meaning of the lure and retrieves other related words (e.g., those that were actually seen at study) or additional semantic information about the lure (e.g., a broader category to which it belongs). Although one might assume that conceptual false recognition must rely on this extensive semantic elaboration process, conceptual true recognition may result from other cognitive processes (e.g., recognition of item-specific details). Furthermore, whereas elaborative processes may often accompany conceptual false recognition, per- ceptual false recognition should not be as dependent on this type of semantic processing; indeed, in the case of perceptual lures, there are no conceptually related words to retrieve from the study lists. Thus, at least some of the frontal activity observed during false compared with true recognition for conceptual (but not perceptual) information likely reflects some combination of the semantic processes previously reported to engage the left frontal cortex (e.g., semantic elaboration, semantic retrieval, etc.). However, further work will be necessary to determine the precise role(s) of the frontal cortex in conceptual false recognition.

Whereas conceptual false recognition may result from more extensive semantic elaboration than conceptual true recognition (as reflected by frontal lobe increases during conceptual false compared with true recognition), perceptual false recognition should occur when the visual and/or phonological features that overlap between the lure and previously studied words are recognized, which may be quite similar to the process of remembering a previously studied word in the case of perceptual true recognition. Indeed, very few regions showed a greater increase in activity during false compared with true recognition for perceptual (but not conceptual) information, which suggests that the neural correlates of false and true perceptual recognition may be more similar than those of conceptual false and true recognition. Moreover, very little activity (i.e., only one cluster bordering the cingulate gyrus) was revealed when perceptual false recognition was directly compared to conceptual false recognition. However, it is important to note that the direct comparison of conceptual and perceptual false recognition was also insensitive to the frontal cortex activity increases during conceptual (but not perceptual) false recognition identified via comparisons involving both false and true recognition. Thus, it is possible that subthreshold activity increases associated with perceptual (but not conceptual) false recognition exist (perhaps within visual or phonological processing regions), but that our current paradigm lacked the power to reveal that differential activity. Future research is necessary to determine whether there are patterns of neural activity that are unique to perceptual false recognition in the same way that certain patterns of frontal cortex activity seem to be unique to conceptual false recognition.

Previous work has suggested that activity in the anterior left inferior frontal cortex (BA 45/47) consistently accompanies conceptual/semantic processing, whereas phonological processing is more often associated with posterior left inferior frontal cortex (BA 44/6) activity (Poldrack et al. 1999; Poldrack and Wagner 2004). In the current study, activity increases in anterior left inferior frontal cortex (BA 45/47) occurred during conceptual (but not perceptual) false compared with true recognition; however, we did not observe posterior inferior frontal cortex activity during perceptual false (compared with true) recognition. As discussed above, one interpretation of these results is that conceptual false recognition may rely on significantly more semantic processing (e.g., elaboration, retrieval of related words) than conceptual true recognition, whereas similar levels of perceptual processing may be necessary for both false and true perceptual recognition. Although our comparisons did not reveal any frontal cortex activity during perceptual false recognition, we did observe posterior left inferior frontal cortex (BA 44/9) activity when we compared all perceptual memory to all conceptual memory, collapsing across accuracy (i.e., true and false perceptual recognition > true and false conceptual recognition). Thus, our results suggest that although perceptual memory in general does benefit more than conceptual memory from the phonological processes supported by the left posterior inferior frontal cortex, perceptual false recognition may not recruit these processes any more than perceptual true recognition does. By contrast, conceptual false recognition does seem to engage the semantic processes sup- 
ported by the left anterior inferior frontal cortex to a greater degree than conceptual true recognition does.

Previous work exploring priming and accurate memory has also identified differential neural activity accompanying the retrieval of conceptual versus perceptual information. In a study of repetition priming, transcranial magnetic stimulation (TMS) was used to disrupt activity in the left frontal cortex during the initial viewings of visual objects (Wig et al. 2005). Subsequent primingrelated activity reductions for those objects were disrupted in left inferior frontal cortex (as were priming-related decreases in response latencies on the behavioral semantic classification task), while neural priming was spared in sensory-processing regions like the middle and inferior occipital cortex. In a study of accurate recollection, multiple regions within the left frontal cortex were preferentially active during the retrieval of conceptual details about previously studied objects, whereas the retrieval of perceptual details of those objects engaged right frontal and bilateral occipito-temporal regions (Dobbins and Wagner 2005). Our results complement these demonstrations that conceptual versus perceptual priming and accurate memory retrieval can rely on distinct neural substrates (specifically that conceptual memory tends to rely on left frontal cortex activity), and we extend these findings to the domain of memory distortion by illustrating that conceptual false recognition is associated with increased left frontal cortex activity. As discussed above, there are several possible explanations for why we did not observe unique patterns of neural activity during perceptual false recognition in the current study. However, given that sensory processing regions in the occipital cortex seem to be preferentially involved in both perceptual priming (Wig et al. 2005) and accurate perceptual retrieval (Dobbins and Wagner 2005), exploring activity in these sensory regions during perceptual false recognition may be an interesting avenue for further investigation.

In summary, although there was substantial overlapping neural activity associated with conceptual and perceptual false recognition compared with correct rejections, conceptual (but not perceptual) false compared with true recognition relied on increased frontal cortex activity (e.g., left inferior and middle frontal cortex), which likely reflected semantic processing. Our results complement previous behavioral work illustrating differential effects of aging and development on conceptual versus perceptual false recognition (Koutstaal et al. 2003; Holliday and Weekes 2006), as well as neuroimaging work reporting preferential engagement of the left frontal cortex in conceptual compared with perceptual priming and accurate retrieval (Dobbins and Wagner 2005; Wig et al. 2005). Moreover, previous research has shown that the neural basis of false recognition can depend on the relatedness of a falsely remembered item to previously studied items (Garoff-Eaton et al. 2006), as well as the context in which one believes to have seen a falsely remembered item (Kahn et al. 2004). Our findings extend this work by indicating that the precise relationship between falsely remembered and previously encountered items can also influence the neural activity that accompanies memory errors. Specifically, this study indicates that the frontal activity that is often observed during false compared with true recognition can be modulated by the relationship between studied and tested items, and it suggests that at least some of this frontal activity may reflect semantic processing during conceptual false recognition, which is distinct from previous arguments that frontal activity during false recognition reflects monitoring processes (e.g., Schacter et al. 1996b; Cabeza et al. 2001).

\section{Materials and Methods}

\section{Participants}

Twenty healthy, right-handed native English speakers with normal or corrected-to-normal vision and no history of serious neu- rological trauma participated in this experiment. Five participants were excluded due to an insufficient number of events in one or more of the conditions of interest, and one additional participant was excluded due to excessive head movement during scanning. Therefore, the results reported here reflect data collected from the remaining 14 participants (mean age, $23.3 \mathrm{yr}$; age range, 18.5-30.9 yr; eight females). Informed consent was acquired from all participants. The Harvard Institutional Review Board approved the behavioral protocol, and the Massachusetts General Hospital Institutional Review Board approved the imaging protocol.

\section{Stimuli}

For this study, 280 groups of seven words were constructed. Each group of seven words consisted of one target (e.g., yell), three words that were conceptually related to that target (e.g., shout, cry, squeal), and three words that were perceptually related to that target (e.g., bell, sell, fell). Words were only considered to be perceptually related to a target if they both rhymed with the target and shared the same spelling as the target (e.g., hear would not be perceptually related to cheer; grown would not be perceptually related to frown). The average word familiarity and Kucera and Francis (K-F) frequency did not differ between the conceptually and perceptually related triplets (both $P>0.2$ ). These seven-word groups were randomly assigned to eight counterbalancing groups. Between these counterbalancing groups, there was no difference in the average familiarity or K-F frequency of the targets, the conceptually related triplets, or the perceptually related triplets (all $P>0.2$ ).

\section{Behavioral procedure}

The scanned memory task was divided into a series of study tasks and recognition tests. In each study task, participants viewed 42 word triplets ( 21 conceptually related triplets and 21 perceptually related triplets) (see Fig. 1). Participants were asked to decide how similar they felt each set of three words were to each other on a scale of one to four (where one was not at all similar and four was extremely similar) and to remember the words for a subsequent recognition test. The word triplets appeared as a vertical word list in the center of the screen. Each set of words was shown for $3 \mathrm{sec}$ followed by a variable duration of fixation ranging from 1-15 sec. Each study task lasted $4 \mathrm{~min} 32 \mathrm{sec}$. Immediately after each study task, participants completed a 1-min 20-sec filler task, which was not scanned. During this task, participants viewed a series of novel, abstract shapes and were asked to rate the pleasantness of each shape. (The filler task was included simply to separate the study task from the recognition test, and participants were told they would not have to remember the shapes from the filler task.) After completing each filler task, participants were given a recognition test consisting of 56 single target words (seven old words that had been studied in a conceptually related word listold conceptual words; seven old words that had been studied in a perceptually related word list-old perceptual words; 14 new words that were related to a conceptually related studied listconceptual lures; 14 new words that were related to a perceptually related studied list-perceptual lures; and 14 new words that were unrelated to any previously studied words-unrelated new words) (see Fig. 1). To assess participants' memory for the targets, we used a modified remember/know/new procedure (Tulving 1985). Participants were asked to respond that they "remembered" a word if they could recall specific details about the event when they studied the word (e.g., the other words that were studied with that word, or where in the list the word had been presented). Participants were instructed to give a "know" response if they knew they saw the word but could not recall specific details about the event when they studied the word. Finally, participants were told to give a "new" response if they did not think they had studied the word. Participants were asked to make the "remember" versus "know" distinction because pilot data indicated that conceptual false alarms were more likely to be accompanied by a "remember" response than were perceptual false alarms. Therefore, had participants simply been asked to make an "old" or 
"new" decision, differences between conceptual and perceptual false memories may have arisen due to differences in the qualitative nature of the retrieved memories. By asking participants to make "remember" versus "know" responses, we were able to compare conceptual to perceptual false recognition for items that were retrieved with the same qualitative nature (i.e., for items associated with a "remember" response).

Each target word was shown for $3 \mathrm{sec}$ followed by a variable duration of fixation ranging from 1-17 sec. Each recognition test lasted 5 min $56 \mathrm{sec}$. Thus, one complete study-test cycle was composed of a study task, filler task, and recognition test. In general, participants completed five full study-test cycles. Data from a single study phase for two participants and a full studytest cycle for one additional participant were excluded because of equipment malfunctions or spiking in the fMRI images. One participant completed only three full study-test cycles due to time constraints.

A particularly important aspect of this paradigm is that a single target can be tested in all five conditions (old conceptual, old perceptual, conceptual lure, perceptual lure, and unrelated new) between participants. All participants were tested on the same set of target words, while the condition in which those words were tested was counterbalanced across participants by manipulating the word triplets shown at study. For each studied word triplet, participants were tested either on an old word from that triplet or on a lure related to that triplet; thus, participants were never tested on multiple words related to the same triplet. In the old conceptual and old perceptual conditions, targets were studied as part of the appropriate (conceptual or perceptual) study triplet, and they were placed in each list position (i.e., top, middle, or bottom) an approximately equal number of times. In the conceptual lure and perceptual lure conditions, targets were not studied, but their appropriate (conceptual or perceptual) study triplets were. And finally, in the unrelated new condition, neither of the study triplets associated with the target was studied. For example, the target word yell could be tested in any of the five conditions by using the following word triplets at study: old conceptual—shout, yell, squeal; old perceptual-fell, bell, yell; conceptual lure-shout, cry, squeal; perceptual lure-fell, bell, sell; unrelated new-no studied triplet.

\section{Imaging methods}

Images were acquired on a three Tesla head-only Siemens Allegra MRI scanner. An angled mirror attached to the head coil reflected images of the stimuli, which were back-projected onto a screen at the superior end of the scanner bore. High resolution anatomical images were acquired using a multiplanar rapidly acquired gradient echo (MP-RAGE) sequence $(\mathrm{TR}=30 \mathrm{msec}$, TE $=3.3 \mathrm{msec}$, 128 slices, $1 \times 1 \times 1.33 \mathrm{~mm}$ voxels). Functional images were acquired using a $\mathrm{T}^{*}$-weighted echo planar imaging (EPI) sequence $(\mathrm{TR}=2000 \mathrm{msec}, \mathrm{TE}=30 \mathrm{msec}$, 34 slices, $4 \times 4 \times 4 \mathrm{~mm}$ voxels). Although images were acquired during both the study tasks and the recognition tests, here we report analyses conducted on images acquired during the recognition tests only.

Unless otherwise noted, SPM99 (Wellcome Department of Cognitive Neurology) was used for all data preprocessing and analysis. Standard preprocessing was performed on the functional data. Images were first slice-time corrected and motion corrected. Then, a temporal high-pass filter was applied to each run to remove linear, quadratic, cubic, and quartic components (using custom software written in MATLAB). Next, all study task runs were concatenated, and all recognition test runs were concatenated. Finally, images were normalized to the SPM99 EPI template (resampling at $2 \times 2 \times 2 \mathrm{~mm}$ resolution) and spatially smoothed (using a 4-mm full-width half-maximum [FWHM] Gaussian kernel).

A general linear model approach was used for the eventrelated analysis. For each participant, activity associated with each event type was modeled using a canonical hemodynamic response function. For the recognition tests, there were 16 possible event types; event types were defined by crossing the three possible response types ("remember," "know," and "new") with the five possible conditions (old conceptual, old perceptual, conceptual lure, perceptual lure, and unrelated new), resulting in 15 event types, with an additional event type defined as any item that received no response. All participants had a minimum of five events in each event type of interest. For each voxel, a $\beta$-weight (i.e., model amplitude) associated with each event type was computed by entering each event type's hemodynamic response model into a general linear model. The difference in magnitude between $\beta$-weights associated with two event types of interest were computed and then compared across participants using one-sample $t$-tests (i.e., a random-effects analysis). Thus, these analyses identified regions that were more active during one event type than another. It is important to note that although it is generally assumed that regions that are more active during a certain task are also more involved in that task, the opposite can also be true (e.g., certain processes, like priming, can be associated with activity decreases) (Schacter and Buckner 1998; Schacter et al. 2007).

Monte Carlo simulations (http://www2.bc.edu/ -slotnics/ scripts.htm) were used to define an individual voxel and cluster extent threshold that would yield results corrected for multiple comparisons (e.g., Forman et al. 1995; Slotnick et al. 2003; Slotnick and Schacter 2004, 2006; Garoff-Eaton et al. 2006). In this study, an individual voxel threshold of $P<0.01$ was used in combination with a cluster extent threshold of 40 resampled voxels, which yielded results corrected for multiple comparisons $(P<0.05)$. For a detailed description of the procedure used to determine these thresholds, see Slotnick and Schacter (2004) or Garoff-Eaton et al. (2006). Spatial autocorrelation was modeled through convolution with a 4-mm FWHM Gaussian kernel in the current study.

In several analyses, to determine whether differential neural activity observed in one contrast was distinct from activity observed in another contrast, we used the masking function in SPM to exclude activity from the second contrast when analyzing the activity from the first. In these analyses, the same voxel and cluster extent thresholds described above $(P<0.01$ and 40 voxels, respectively) were used for both the excluded contrast and the contrast of interest. Voxel coordinates are reported in Talairach space (Talairach and Tournoux 1988) and refer to the most significant voxel proximal to the center of each active region.

Event-related timecourses were extracted from active clusters of interest in order to characterize the nature of the neural activity in those regions (for details, see Slotnick and Schacter 2004). Using custom software written in MATLAB, linear trends were extracted from the timecourses. Statistics were performed on the mean event-related activity occurring 4-8 sec after stimulus onset unless otherwise noted, and activity occurring 0-4 sec before stimulus onset was used to correct for baseline activity. Given that these timecourses were used in post hoc analyses characterizing the neural activity in clusters defined by the random effects analyses, one-tailed $t$-tests were used to compare timecourses associated with different event types within those active clusters. To compute the joint probability of observing a specific pattern of activity (i.e., the same relationship between timecourses associated with two event types) across multiple regions of interest, we used Fisher's technique (Fisher 1973), as described by Slotnick and Schacter (2006).

\section{Acknowledgments}

We thank Scott D. Slotnick for writing the MATLAB scripts used to concatenate the functional imaging data and to extract the event-related timecourses. This research was supported by NIMH grant MH060941 (to D.L.S.) and an NSF Graduate Research Fellowship (to R.J.G.-E.).

\section{References}

Achim, A.M. and Lepage, M. 2005. Dorsolateral prefrontal cortex involvement in memory Postretrieval monitoring revealed in both item and associative recognition tests. Neuroimage 24: 1113-1121. Anderson, N.D. and Craik, F.I.M. 2000. Memory in the aging brain. In The Oxford handbook of memory (eds. E. Tulving and F.I.M. Craik), pp. 
411-425. Oxford University Press, New York.

Buckner, R.L. and Wheeler, M.E. 2001. The cognitive neuroscience of remembering. Nat. Rev. Neurosci. 2: 624-634.

Buckner, R.L., Koutstaal, W., Schacter, D.L., Wagner, A.D., and Rosen, B.R. 1998. Functional-anatomic study of episodic retrieval using fMRI. Neuroimage 7: 151-162.

Budson, A.E., Sullivan, A.L., Daffner, K.R., and Schacter, D.L. 2003. Semantic versus phonological false recognition in aging and Alzheimer's disease. Brain Cogn. 51: 251-261.

Cabeza, R., Rao, S.M., Wagner, A.D., Mayer, A.R., and Schacter, D.L. 2001. Can medial temporal lobe regions distinguish true from false? An event-related functional MRI study of veridical and illusory recognition memory. Proc. Natl. Acad. Sci. 98: 4805-4810.

Cabeza, R., Locantore, J.K., and Anderson, N.D. 2003. Lateralization of prefrontal activity during episodic memory retrieval: Evidence for the production-monitoring hypothesis. J. Cogn. Neurosci. 15: $249-259$.

Demb, J.B., Desmond, J.E., Wagner, A.D., Vaidya, C.J., Glover, G.H., and Gabrieli, J.D. 1995. Semantic encoding and retrieval in the left inferior prefrontal cortex: A functional MRI study of task difficulty and process specificity. J. Neurosci. 15: $5870-5878$.

Diamond, A. 2002. Normal development of prefrontal cortex from birth to young adulthood: cognitive functions, anatomy, and biochemistry. In Principles of frontal lobe function (eds. D.T. Stuss and R.T. Knight), pp. 466-503. Oxford University Press, New York.

Dobbins, I.G. and Wagner, A.D. 2005. Domain-general and domain-sensitive prefrontal mechanisms for recollecting events and detecting novelty. Cereb. Cortex 15: 1768-1778.

Dobbins, I.G., Simons, J.S., and Schacter, D.L. 2004. fMRI evidence for separable and lateralized prefrontal memory monitoring processes. $J$. Cogn. Neurosci. 16: 908-920.

Fisher, R.A. 1973. Statistical methods for research workers, 14th ed. Hafner Publishing Company, New York.

Forman, S.D., Cohen, J.D., Fitzgerald, M., Eddy, W.F., Mintun, M.A., and Noll, D.C. 1995. Improved assessment of significant activation in functional magnetic resonance imaging (fMRI): Use of a cluster-size threshold. Magn. Reson. Med. 33: 636-647.

Garoff-Eaton, R.J., Slotnick, S.D., and Schacter, D.L. 2006. Not all false memories are created equal: The neural basis of false recognition. Cereb. Cortex 16: 1645-1652.

Goldmann, R.E., Sullivan, A.L., Droller, D.B., Rugg, M.D., Curran, T., Holcomb, P.J., Schacter, D.L., Daffner, K.R., and Budson, A.E. 2003. Late frontal brain potentials distinguish true and false recognition. Neuroreport 14: 1717-1720.

Gonsalves, B., Reber, P.J., Gitelman, D.R., Parrish, T.B., Mesulam, M.M., and Paller, K.A. 2004. Neural evidence that vivid imagining can lead to false remembering. Psychol. Sci. 15: 655-660.

Henson, R.N., Shallice, T., and Dolan, R.J. 1999. Right prefrontal cortex and episodic memory retrieval: A functional MRI test of the monitoring hypothesis. Brain 122: 1367-1381.

Holliday, R.E. and Weekes, B.S. 2006. Dissociated developmental trajectories for semantic and phonological false memories. Memory 14: 624-636.

Johnson, M.K., Hashtroudi, S., and Lindsay, D.S. 1993. Source monitoring. Psychol. Bull. 114: 3-28.

Johnson, M.K., Nolde, S.F., Mather, M., Kounios, J., Schacter, D.L., and Curran, T. 1997. The similarity of brain activity associated with true and false recognition memory depends on test format. Psychol. Sci. 8: $250-257$.

Kahn, I., Davachi, L., and Wagner, A.D. 2004. Functionalneuroanatomic correlates of recollection: Implications for models of recognition memory. J. Neurosci. 24: 4172-4180.

Kensinger, E.A. and Schacter, D.L. 2005. Emotional content and reality-monitoring ability: fMRI evidence for the influences of encoding processes. Neuropsychologia 43: 1429-1443.

Kirchhoff, B.A., Schapiro, M.L., and Buckner, R.L. 2005. Orthographic distinctiveness and semantic elaboration provide separate contributions to memory. J. Cogn. Neurosci. 17: 1841-1854.

Koutstaal, W., Reddy, C., Jackson, E.M., Prince, S., Cendan, D.L., and Schacter, D.L. 2003. False recognition of abstract versus common objects in older and younger adults: Testing the semantic categorization account. J. Exp. Psychol. Learn. Mem. Cogn. 29: $499-510$.

Loftus, E.F. 2003. Make-believe memories. Am. Psychol. 58: 867-873.

Okado, Y. and Stark, C.E. 2005. Neural activity during encoding predicts false memories created by misinformation. Learn. Mem. 12: 3-11.

Parkin, A.J., Bindschaedler, C., Harsent, L., and Metzler, C. 1996.
Pathological false alarm rates following damage to the left frontal cortex. Brain Cogn. 32: 14-27.

Petersen, S.E., Fox, P.T., Posner, M.I, Mintun, M., and Raichle, M.E. 1988. Positron emission tomographic studies of the cortical anatomy of single-word processing. Nature 331: 585-589.

Poldrack, R.A. and Wagner, A.D. 2004. What can neuroimaging tell us about the mind? Insights from prefrontal cortex. Curr. Dir. Psychol. Sci. 13: $177-181$.

Poldrack, R.A., Wagner, A.D., Prull, M.W., Desmond, J.E., Glover, G.H., and Gabrieli, J.D. 1999. Functional specialization for semantic and phonological processing in the left inferior prefrontal cortex. Neuroimage 10: 15-35.

Prince, S.E., Daselaar, S.M., and Cabeza, R. 2005. Neural correlates of relational memory: successful encoding and retrieval of semantic and perceptual associations. J. Neurosci. 25: 1203-1210.

Raz, N. 2000. Aging of the brain and its impact on cognitive performance: Integration of structural and functional findings. In The handbook of aging and cognition (eds. F.I.M. Craik and T.A. Salthouse), pp. 1-90. Lawrence Erlbaum Associates Publishers, Mahwah, NJ.

Roediger III, H.L. and McDermott, K.B. 1995. Creating false memories: Remembering words not presented in lists. J. Exp. Psychol. Learn. Mem. Cogn. 21: 803-814.

Rugg, M.D., Fletcher, P.C., Frith, C.D., Frackowiak, R.S., and Dolan, R.J. 1996. Differential activation of the prefrontal cortex in successful and unsuccessful memory retrieval. Brain 119: 2073-2083.

Rugg, M.D., Henson, R.N., and Robb, W.G. 2003. Neural correlates of retrieval processing in the prefrontal cortex during recognition and exclusion tasks. Neuropsychologia 41: 40-52.

Schacter, D.L. and Buckner, R.L. 1998. Priming and the brain. Neuron 20: $185-195$.

Schacter, D.L. and Slotnick, S.D. 2004. The cognitive neuroscience of memory distortion. Neuron 44: 149-160.

Schacter, D.L., Curran, T., Galluccio, L., Milberg, W.P., and Bates, J.F. 1996a. False recognition and the right frontal lobe: A case study. Neuropsychologia 34: 793-808.

Schacter, D.L., Reiman, E., Curran, T., Yun, L.S., Bandy, D., McDermott, K.B., and Roediger III, H.L. 1996b. Neuroanatomical correlates of veridical and illusory recognition memory: Evidence from positron emission tomography. Neuron 17: 267-274.

Schacter, D.L., Buckner, R.L., Koutstaal, W., Dale, A.M., and Rosen, B.R. 1997a. Late onset of anterior prefrontal activity during true and false recognition: An event-related fMRI study. Neuroimage 6: 259-269.

Schacter, D.L., Verfaellie, M., and Anes, M.D. 1997b. Illusory memories in amnesic patients: Conceptual and perceptual false recognition. Neuropsychology 11: 331-342.

Schacter, D.L., Wig, G.S., and Stevens, W.D. 2007. Reductions in cortical activity during priming. Curr. Opin. Neurobiol. 17: 171-176.

Slotnick, S.D. and Schacter, D.L. 2004. A sensory signature that distinguishes true from false memories. Nat. Neurosci. 7: 664-672.

Slotnick, S.D. and Schacter, D.L. 2006. The nature of memory related activity in early visual areas. Neuropsychologia 44: 2874-2886.

Slotnick, S.D., Moo, L.R., Segal, J.B., and Hart Jr., J. 2003. Distinct prefrontal cortex activity associated with item memory and source memory for visual shapes. Brain Res. Cogn. Brain Res. 17: 75-82.

Swick, D. and Knight, R.T. 1999. Contributions of prefrontal cortex to recognition memory: Electrophysiological and behavioral evidence. Neuropsychology 13: 155-170.

Talairach, J. and Tournoux, P. 1988. Co-planar stereotaxic atlas of the human brain. Thieme, New York.

Tulving, E. 1985. Memory and consciousness. Can. Psychol. 26: 1-12.

Wagner, A.D., Paré-Blagoev, E.J., Clark, J., and Poldrack, R.A. 2001. Recovering meaning: Left prefrontal cortex guides controlled semantic retrieval. Neuron 31: 329-338.

Watson, J.M., Balota, D.A., and Roediger III, H.L. 2003. Creating false memories with hybrid lists of semantic and phonological associates: Over-additive false memories produced by converging associative networks. J. Mem. Lang. 49: 95-118.

Wheeler, M.E. and Buckner, R.L. 2004. Functional-anatomic correlates of remembering and knowing. Neuroimage 21: 1337-1349.

Wig, G.S., Grafton, S.T., Demos, K.E., and Kelley, W.M. 2005 Reductions in neural activity underlie behavioral components of repetition priming. Nat. Neurosci. 8: 1228-1233.

Received July 7, 2007; accepted in revised form August 10, 2007. 


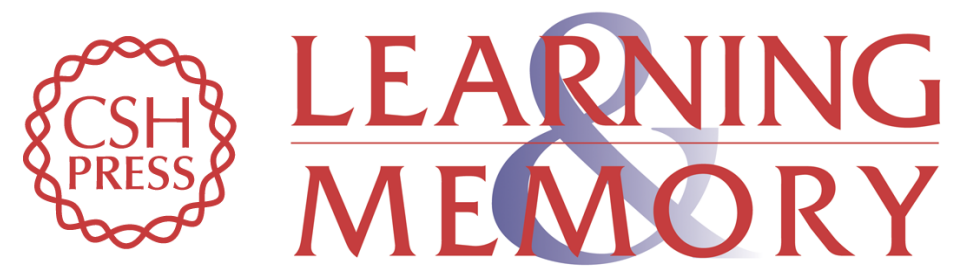

\section{The neural correlates of conceptual and perceptual false recognition}

Rachel J. Garoff-Eaton, Elizabeth A. Kensinger and Daniel L. Schacter

Learn. Mem. 2007, 14:

Access the most recent version at doi:10.1101//m.695707

References This article cites 47 articles, 5 of which can be accessed free at:

http://learnmem.cshlp.org/content/14/10/684.full.html\#ref-list-1

License

Email Alerting Receive free email alerts when new articles cite this article - sign up in the box at the Service top right corner of the article or click here. 